\title{
Quarkonium-Hadron Interactions in QCD
}

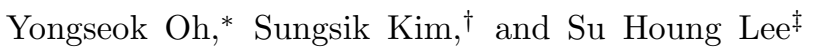 \\ Institute of Physics and Applied Physics, Department of Physics, Yonsei University, Seoul 120-749, Korea
}

\begin{abstract}
The leading order non-relativistic QCD result for the quarkonium-hadron interaction cross section is derived by using the QCD factorization formula combined with the Bethe-Salpeter amplitude for the heavy quark bound state. Our result shows the equivalence of this approach with the operator product expansion even for the bound state scattering to leading order in QCD. We also apply the formula to the charmonium system and investigate the relativistic correction coming from the phase space of the reaction. We find that although $J / \psi$-gluon cross-section gets a large relativistic correction at higher energies, the correction becomes small in the $J / \psi$-hadron cross section due to the increase of the gluon distribution at smaller $x$ inside the hadron.

PACS numbers: 25.75.-q, 12.38.-t, 13.75.-n, 13.85.Lg
\end{abstract}

More than two decades ago, Peskin [1] and Bhanot and Peskin [2] showed that one could apply perturbative Quantum Chromodynamics (pQCD) to calculate the interactions between the bound state of heavy quarks and the light hadrons. Such calculation was feasible because in the large quark mass limit one could consistently obtain the leading order operator product expansion (OPE) of the correlation function between two heavy meson currents in the light hadron state. The justification of such calculation stems from the fact that there exist two relevant scales in the bound state [3] in the large quark mass limit, namely the binding energy, which becomes Coulomb-like and scales as $m g^{4}$, and the typical momentum scale of the bound state, which scales like $m g^{2}$, where $m$ is the heavy quark mass and $g$ the quark-gluon coupling constant. Hence, taking the separation scale of the OPE to be the binding energy, it is consistent to take into account the bound state property, which is generated by the typical momentum scale of the bound state, into the process-dependent Wilson coefficient.

The calculation is challenging in itself, and has attracted recent interests because when applied to the $J / \psi$ system [4, 5], the strength of the $J / \psi$-hadron inelastic scattering cross section determines the amount of the $J / \psi$ suppression [6] in heavy ion collisions due to hadronic final state interactions [7]. Moreover, other existing model calculations for $\sigma_{J / \psi-h}$, based on meson exchange models [8], quark exchange models [9], or QCD sum rules 110] give very different energy dependence and magnitude near threshold, which shows the importance of the nonperturbative effects. Therefore, more careful analyses of each calculation are necessary, where the limitations and expected corrections would be explicitly spelled out.

In this paper, we will derive the leading order pQCD result using the QCD factorization theorem. The result obtained by Peskin and Bhanot was derived within the OPE [1, 2]. The equivalence between the OPE and the factorization approach is well established in the deep inelastic scattering and Drell-Yan processes [11]. As will be shown here, the equivalence between the two approaches are also true for bound state scattering to leading order in QCD. Similar approach has been used by one of us (SHL) to estimate the dissociation cross section of the $J / \psi$ at finite temperature [12]. The factorization formula also provides a manageable starting point to calculate higher twist gluonic effects [13, 14], which should be nontrivial for the $J / \psi$-hadron scattering.

We refer to a bound state of heavy quark and its own antiquark as $\Phi$. According to the factorization formula, the total scattering cross section of $\Phi$ with a hadron $h$ can be written as 2 , 15]

$$
\sigma_{\Phi h}(\nu)=\int_{0}^{1} d x \sigma_{\Phi g}(x \nu) g(x)
$$

with $\nu=p \cdot q / M_{\Phi}$, where $p(q)$ is the momentum of the hadron $(\Phi)$ and $M_{\Phi}$ is the $\Phi$ mass. Here, $\sigma_{\Phi g}$ is the perturbative $\Phi$-gluon scattering cross section and $g(x)$ is the leading twist gluon distribution function within the hadron. The separation scale is taken to be the binding energy of the bound state. Hence, the bound state properties have to be taken into account in $\sigma_{\Phi g}$. This can be accomplished by introducing the Bethe-Salpeter (BS) amplitude $\Gamma\left(p_{1},-p_{2}\right)$, which satisfies 16 .

$$
\begin{gathered}
\Gamma_{\mu}\left(p_{1},-p_{2}\right)=i C_{\text {color }} \int \frac{d^{4} k}{(2 \pi)^{4}} g^{2} V(k) \gamma^{\nu} \Delta\left(p_{1}+k\right) \\
\times \Gamma_{\mu}\left(p_{1}+k,-p_{2}+k\right) \Delta\left(-p_{2}+k\right) \gamma_{\nu},
\end{gathered}
$$

where $C_{\text {color }}=\left(N_{c}^{2}-1\right) /\left(2 N_{c}\right)$ with the number of color $N_{c}$. The trivial color indices have been suppressed. Figure 1 shows the diagrammatic representation of the BS

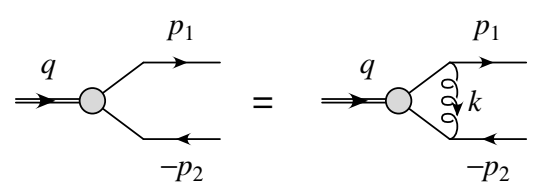

FIG. 1: The Bethe-Salpeter equation for the quarkonium $\Phi$. 
equation, where $p_{1}\left(-p_{2}\right)$ is the four-momentum of the heavy quark (anti-quark) with $\Delta(p)=1 /(\not p-m+i \epsilon)$ and $V(k)=-1 /\left(k^{2}+i \epsilon\right)$.

We introduce $\phi_{\mu}$, which is defined as

$$
\phi_{\mu}(q, \mathbf{p}) \equiv\left(\frac{N_{c}}{M_{\Phi}}\right)^{1 / 2} \int \frac{d p_{0}}{2 \pi} \Delta\left(p_{1}\right) \Gamma_{\mu}\left(p_{1},-p_{2}\right) \Delta\left(-p_{2}\right)
$$

where $q=p_{1}+p_{2}$ and $p=\left(p_{1}-p_{2}\right) / 2$. We work in the $\Phi$ rest frame and introduce the binding energy $\varepsilon$, such that $q_{0}=M_{\Phi}=2 m+\varepsilon(\varepsilon<0)$. Then, in the non-relativistic limit, Eq. (3) reduces to

$$
\begin{aligned}
\phi_{\mu}(q, \mathbf{p})= & \frac{-i}{\varepsilon-\mathbf{p}^{2} / m} \sqrt{\frac{N_{c}}{M_{\Phi}}} \frac{1+\gamma_{0}}{2} \\
& \times \Gamma_{\mu}\left(\frac{q}{2}+p,-\frac{q}{2}+p\right) \frac{1-\gamma_{0}}{2},
\end{aligned}
$$

where $p_{0}=\varepsilon / 2-\mathbf{p}^{2} /(2 m)$. In this limit, the BS amplitude reduces to

$$
\begin{aligned}
& \Gamma_{\mu}(q / 2+p,-q / 2+p) \\
= & -\left(\varepsilon-\frac{\mathbf{p}^{2}}{m}\right) \sqrt{\frac{M_{\Phi}}{N_{c}}} \psi(\mathbf{p}) \frac{1+\gamma_{0}}{2} \gamma_{i} \delta_{\mu i} \frac{1-\gamma_{0}}{2},
\end{aligned}
$$

and the corresponding BS equation becomes the nonrelativistic Schrödinger equation for the Coulombic bound state,

$$
\left(\varepsilon-\frac{\mathbf{p}^{2}}{m}\right) \psi(\mathbf{p})=-g^{2} C_{\text {color }} \int \frac{d^{3} k}{(2 \pi)^{3}} V(\mathbf{k}) \psi(\mathbf{p}+\mathbf{k}),
$$

so that $\psi(\mathbf{p})$ is the normalized wave function for the bound state. For the pseudo scalar bound state, we replace $\gamma_{i} \delta_{\mu i}$ to $\gamma_{5}$ in Eq. (5), but obtain the same spatial wave function. (Some discussions on the realistic potential can be found, for example, in Ref. [17].) It is straightforward to verify that our formalism gives the well-known result for the $\Phi \rightarrow e^{+} e^{-}$decay width, $\Gamma\left(\Phi \rightarrow e^{+} e^{-}\right)=$ $\frac{16 \pi \alpha_{\mathrm{em}}^{2} Q_{h}^{2} N_{c}}{3 M_{\Phi}^{2}}|\psi(r=0)|^{2}$, where $\alpha_{\mathrm{em}}=e^{2} /(4 \pi)$ and $Q_{h}$ is the heavy quark charge in the unit of $e$.

With the BS amplitude defined as Eq. (5), we now obtain the $\Phi$-gluon scattering amplitude with the processes depicted in Fig. 2. The scattering matrix elements can be written as $\mathcal{M}=\varepsilon_{\mu}(\Phi) \mathcal{M}^{\mu \nu} \varepsilon_{\nu}(g)$, where $\varepsilon_{\mu}(\Phi)$ and

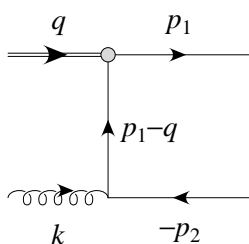

(a)

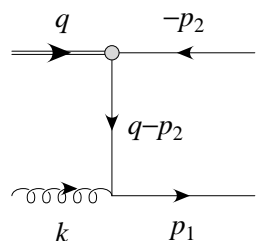

(b)

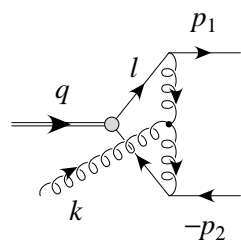

(c)
FIG. 2: Scattering processes of $\Phi$ and gluon.

$\varepsilon_{\nu}(g)$ are the polarization vectors of the $\Phi$ and the gluon, respectively. We first compute $\mathcal{M}_{1}^{\mu \nu}$, which comes from Figs. 2( $(\mathrm{a}, \mathrm{b})$,

$$
\begin{aligned}
\mathcal{M}_{1}^{\mu \nu}= & -g \bar{u}\left(p_{1}\right)\left[\Gamma^{\mu}\left(p_{1},-q+p_{1}\right) \Delta\left(-q+p_{1}\right) \gamma^{\nu}\right. \\
& \left.+\gamma^{\nu} \Delta\left(q-p_{2}\right) \Gamma^{\mu}\left(q-p_{2},-p_{2}\right)\right] T^{a} v\left(p_{2}\right),
\end{aligned}
$$

where $u(p)$ and $v(p)$ are the Dirac spinors of the quark and antiquark, respectively, $a$ is the color index of the incoming gluon, and $T^{a}$ is the color matrix with $\operatorname{Tr}\left(T^{a} T^{b}\right)=\delta^{a b} / 2$. To obtain the leading order nonrelativistic result, we have to discuss the counting scheme in the rest frame of $\Phi$. First the binding energy $\varepsilon_{0}=$ $m\left[N_{c} g^{2} /(16 \pi)\right]^{2}$ is $O\left(m g^{4}\right)$. Combined with the energy conservation $q+k=p_{1}+p_{2}$ in the non-relativistic limit this implies the following counting,

$$
\left|\mathbf{p}_{\mathbf{1}}\right| \sim\left|\mathbf{p}_{\mathbf{2}}\right| \sim O\left(m g^{2}\right), \quad k^{0}=|\mathbf{k}| \sim O\left(m g^{4}\right) .
$$

Then the leading order result of Eq. (7) reads

$$
\begin{aligned}
\mathcal{M}_{1}^{\mu \nu}= & g \sqrt{\frac{M_{\Phi}}{N_{c}}}\left\{-\mathbf{k} \cdot \frac{\partial \psi(\mathbf{p})}{\partial \mathbf{p}} \delta^{\nu 0}+\frac{2 p^{j}}{m} \psi(\mathbf{p}) \delta^{\nu j}\right\} \delta^{\mu i} \\
& \times \bar{u}\left(p_{1}\right) \frac{1+\gamma_{0}}{2} \gamma^{i} T^{a} \frac{1-\gamma_{0}}{2} v\left(p_{2}\right) .
\end{aligned}
$$

Figure 2(c) gives

$$
\begin{aligned}
\mathcal{M}_{2}^{\mu \nu}= & g^{3} f_{a b c} T^{c} T^{b} \bar{u}\left(p_{1}\right) \int \frac{d^{4} l}{(2 \pi)^{4}} V\left(l-p_{1}\right) V\left(l-p_{1}+k\right) \gamma_{\beta} \Delta(l) \Gamma^{\mu}(l, l-q) \Delta(l-q) \gamma_{\alpha} v\left(p_{2}\right) \\
& \times\left[g^{\alpha \beta}\left(2 l-2 p_{1}+k\right)^{\nu}+g^{\beta \nu}\left(p_{1}-l+k\right)^{\alpha}-g^{\nu \alpha}\left(l-p_{1}+2 k\right)^{\beta}\right] .
\end{aligned}
$$

The color factor of this diagram is $f_{a b c} T^{c} T^{b}=-\frac{i}{2} N_{c} T^{a}$. There is one more diagram, where the external gluon leg is attached to the internal quark line within the inter- nal loop. However such a diagram carries the color factor $T^{b} T^{a} T^{b}=-T^{a} /\left(2 N_{c}\right)$ (color adjoint potential) and hence is suppressed by $1 / N_{c}^{2}$ compared to Fig. 2(c) [1]. 
Using the BS equation and the counting (8), we get

$$
\begin{aligned}
M_{2}^{\mu \nu}= & -g \sqrt{\frac{M_{\Phi}}{N_{c}}}\left\{k_{0} \frac{\partial \psi(\mathbf{p})}{\partial p^{j}}+\frac{2 p^{j}}{m} \psi(\mathbf{p})\right\} \delta^{\mu i} \delta^{\nu j} \\
& \times \bar{u}\left(p_{1}\right) \frac{1+\gamma_{0}}{2} \gamma^{i} \frac{1-\gamma_{0}}{2} T^{a} v\left(p_{2}\right) .
\end{aligned}
$$

Collecting Eqs. (9) and (11) gives the (gauge invariant) leading order result for the scattering amplitude as

$$
\begin{aligned}
\mathcal{M}^{\mu \nu}= & -g \sqrt{\frac{M_{\Phi}}{N_{c}}}\left\{\mathbf{k} \cdot \frac{\partial \psi(\mathbf{p})}{\partial \mathbf{p}} \delta^{\nu 0}+k_{0} \frac{\partial \psi(\mathbf{p})}{\partial p^{j}} \delta^{\nu j}\right\} \delta^{\mu i} \\
& \times \bar{u}\left(p_{1}\right) \frac{1+\gamma_{0}}{2} \gamma^{i} \frac{1-\gamma_{0}}{2} T^{a} v\left(p_{2}\right) .
\end{aligned}
$$

The scattering cross section $\sigma_{\Phi g}$ can be obtained from

$$
\begin{aligned}
\sigma_{\Phi g} & =\int \frac{1}{4 M_{\Phi} k_{0}} \overline{|\mathcal{M}|^{2}}(2 \pi)^{4} \delta^{4}\left(p_{1}+p_{2}-k-q\right) \frac{d^{3} p_{1}}{2 p_{1}^{0}(2 \pi)^{3}} \frac{d^{3} p_{2}}{2 p_{2}^{0}(2 \pi)^{3}} \\
& =\int \frac{\left|\mathbf{p}_{1}\right|^{3}}{M_{\Phi}\left(k_{0} p_{1}^{0}-m^{2}\right)+m^{2}\left(p_{1}^{0}-k_{0}\right)+p_{1}^{0}\left(M_{\Phi}^{2}-2 m^{2}\right) / 2} \frac{\overline{\left.\mathcal{M}\right|^{2}}}{64 \pi^{2} M_{\Phi} k_{0}} d \Omega_{\mathrm{lab}} \\
& \approx \int \frac{\sqrt{k_{0}+\varepsilon}}{128 \pi^{2} M_{\Phi} k_{0} \sqrt{m}} \overline{|\mathcal{M}|^{2}} d \Omega_{\mathrm{lab}}
\end{aligned}
$$

in the $\Phi$ rest frame, where $\overline{|\mathcal{M}|^{2}}=\frac{1}{6\left(N_{c}^{2}-1\right)} \sum|\mathcal{M}|^{2}$ and the average is over the initial polarizations and the color of the gluon. Note that going from the second line to the third line in Eq. (13), we use the non-relativistic approximation (8). With the amplitude (12) we obtain

$$
\overline{|\mathcal{M}|^{2}}=\frac{4 g^{2} m^{2} M_{\Phi} k_{0}^{2}}{3 N_{c}}|\nabla \psi(\mathbf{p})|^{2}
$$

Making use of the ground state wave function of the Coulombic bound state,

$$
\boldsymbol{\nabla} \psi_{1 S}(\mathbf{k})=-i a_{0}^{5 / 2} 32 \sqrt{\pi} \frac{a_{0} \mathbf{k}}{\left[\left(a_{0} k\right)^{2}+1\right]^{3}},
$$

where $\varepsilon=-\varepsilon_{0}$ and $a_{0}=16 \pi /\left(g^{2} N_{c} m\right)$, we finally obtain

$$
\sigma_{\Phi g}(\lambda)=\frac{128 g^{2}}{3 N_{c}} a_{0}^{2} \frac{\left(\lambda / \varepsilon_{0}-1\right)^{3 / 2}}{\left(\lambda / \varepsilon_{0}\right)^{5}}
$$

with $\lambda=q \cdot k / M_{\Phi}$. Hence, we have derived anew the result for $\sigma_{\Phi g}$ of Ref. [2]. The scattering cross section $\sigma_{\Phi h}$ can then be computed using the factorization formula (11).

To apply the same procedure to the scattering of $\Phi^{\prime}$ (the $2 S$ state), we use the wave function

$$
\boldsymbol{\nabla} \psi_{2 S}(\mathbf{k})=-i a_{0}^{5 / 2} 8 \sqrt{2 \pi} \frac{\left[\left(a_{0} k\right)^{2}-\frac{1}{2}\right]}{\left[\left(a_{0} k\right)^{2}+\frac{1}{4}\right]^{4}} a_{0} \mathbf{k}
$$

with the binding energy $\varepsilon=-\varepsilon_{0} / 4$. This then leads to

$$
\sigma_{\Phi^{\prime} g}=\frac{16 g^{2}}{N_{c}^{2}} a_{0}^{2} \frac{\left(\lambda / \varepsilon_{0}-\frac{1}{4}\right)^{3 / 2}\left(\lambda / \varepsilon_{0}-\frac{3}{4}\right)^{2}}{\left(\lambda / \varepsilon_{0}\right)^{7}}
$$

which is consistent with the result of Ref. [5]. It should be noted, however, that the application of our formalism should be less reliable for the excited states becasue the

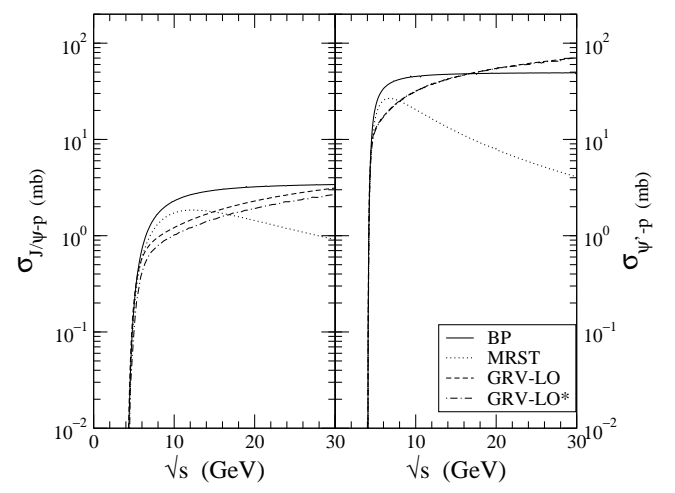

FIG. 3: Scattering cross sections $\sigma_{J / \psi-p}$ and $\sigma_{\psi^{\prime}(2 S)-p}$. The solid lines are obtained with $g(x)$ of Ref. [2] (BP), while the dashed and dotted lines are with that of Ref. 18 (MRST) and 19] (GRV-LO), respectively. The cross sections with relativistic corrections are given by dot-dashed lines with $g(x)$ of Ref. 19 (GRV-LO*).

wave function would be more affected by the confining part of the potential.

We now plot the numerical results for the $\sigma_{\Phi p}$ and $\sigma_{\Phi^{\prime} p}$ in Fig. 3. Similar plot has been already given in Refs. [4, 5], but here we use the original parameters as given in Ref. [2]: $\varepsilon_{0}=780 \mathrm{MeV}$ and $m=1.95 \mathrm{GeV}$ as determined in Ref. [2] by fitting the $J / \psi$ and $\psi^{\prime}(2 S)$ masses to a Coulombic spectrum. Also given in Fig. 3 are the results with the relativistic correction (dot-dashed lines) that will be discussed below. For the gluon distribution function of the proton, we use the parameterization of Ref. [2], $g(x)=0.5(\eta+1)(1-x)^{\eta} / x$ with $\eta=5(\mathrm{BP})$ as well as the MRST [18] and the GRV-LO gluon distributions 19. The results show the dependence of the cross sections on the gluon distribution function. With the 
distribution functions of Refs. [2, 19], the cross sections increase with the center-of-mass energy $\sqrt{s}$. But $g(x)$ of Ref. [18] leads to the decrease of the cross sections at higher energies, which comes from the difference in $g(x)$ at small $x$. Looking at the ratio $\sigma_{\psi^{\prime}(2 s)-p} / \sigma_{J / \psi-p}$ within this approach, one finds that it has a peak at $\sqrt{s} \approx 4.2$ $\mathrm{GeV}$. The peak value is about $2000 \sim 5000$ but it quickly converges to $4 \sim 20$ at higher energies depending on the form of $g(x)$. In a quark exchange model the ratio between the maxima of the cross sections was estimated to be around 6 [9].

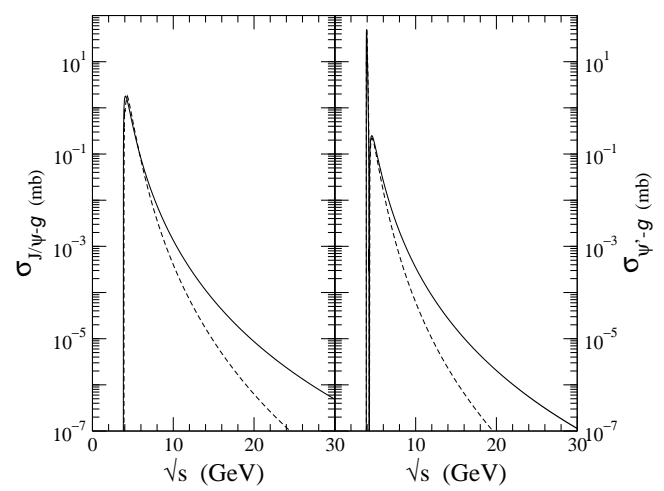

FIG. 4: Scattering cross sections $\sigma_{J / \psi-g}$ and $\sigma_{\psi^{\prime}(2 S)-g}$. The solid lines are obtained in the non-relativistic limit and the dashed lines are with the relativistic correction.

Let us now consider the relativistic corrections to our results. The non-relativistic counting scheme (8) of this formalism implies that the formula (16) should not be used at high gluon energy. However, calculating relativistic corrections in this formalism is intricately related to the higher $g^{2}$ corrections, which is a formidable future task. Here, we will only look at the simple but important relativistic correction coming from the relativistic calculation of the phase space integral in Eq. (13). The difference after a full calculation is shown in Fig. 1 for $\sigma_{\Phi g}$, which shows larger suppression of the cross sections at higher energy. In order to see the relativistic corrections in $\sigma_{\Phi p}$ we compute $\sigma_{\Phi p}$ with the $\sigma_{\Phi g}$ after the relativistic correction using the GRV-LO gluon distribution function (the dot-dashed lines in Fig. 3). We find that this relativistic correction does not have much effect on the cross section $\sigma_{\Phi h}$. This follows because $\sigma_{\Phi g}$ is highly peaked at small energy region and the gluon distribution function increases towards small $x$. Through Eq. (11), this implies that $\sigma_{\Phi h}$, at all energies, are dominated by the low energy behavior of $\sigma_{\Phi g}$, which is reliably calculated in the non-relativistic limit. In fact, it has been observed in a potential model that the relativistic treatment of the bound state wave functions does not improve much the nonrelativistic description 20]. All these imply that the full relativistic corrections to the scattering amplitude $\sigma_{\Phi g}$ would have little effects on $\sigma_{\Phi h}$.

The remaining important and interesting correction is the higher twist-effect, which contributes as $A / \varepsilon^{2}$ in our formalism. Since $A$ is related to some hadronic scale of the hadron $h$, it should be of the order $\varepsilon^{2}$ itself, hence should be non-negligible. The research in this direction awaits more experimental and theoretical works [13, 14 . It is also important to extend this calculation to investigate the absortion cross section for the $\chi$ states. This will influence the amount of $J / \psi$ production coming from the decay of the $\chi$ 's in a p-A or A-A reaction [21].

We are grateful to A. Hayashigaki and C.-Y. Wong for fruitful discussions. This work was supported in part by the Brain Korea 21 project of Korean Ministry of Education, KOSEF under Grant No. 1999-2-111-005-5, the Yonsei University Research Grant, and the Korean Ministry of Education under Grant No. 2000-2-0689.

* Electronic address: yoh@phya.yonsei.ac.kr

† Electronic address: sskim@phya.yonsei.ac.kn; Present address: Department of Physics, Sungkyunkwan University, Suwon 440-746, Korea

‡ Electronic address: suhoung@phya.yonsei.ac.kr

[1] M. E. Peskin, Nucl. Phys. B156, 365 (1979).

[2] G. Bhanot and M. E. Peskin, Nucl. Phys. B156, 391 (1979).

[3] T. Appelquist, M. Dine, and I. Muzinich, Phys. Rev. D 17, 2074 (1978).

[4] D. Kharzeev and H. Satz, Phys. Lett. B 334, 155 (1994); D. Kharzeev et al., ibid. 389, 595 (1996).

[5] F. Arleo et al., Phys. Rev. D 65, 014005 (2002).

[6] T. Matsui and H. Satz, Phys. Lett. B 178, 416 (1986).

[7] R. Vogt, Phys. Rep. 310, 197 (1999).

[8] S. G. Matinyan and B. Müller, Phys. Rev. C 58, 2994 (1998); K. L. Haglin, ibid. 61, 031902 (2000); Z. Lin and C. M. Ko, ibid. 62, 034903 (2000); Y. Oh, T. Song, and Su H. Lee, ibid. 63, 034901 (2001); A. Sibirtsev, K. Tsushima, and A. W. Thomas, ibid. 63, 044906 (2001); W. Liu, C. M. Ko, and Z. W. Lin, nucl-th/0107058,

[9] C.-Y. Wong, E. S. Swanson, and T. E. Barnes, Phys. Rev. C 62, 045201 (2000); 65, 014903 (2002); C.-Y. Wong, ibid. 65, 034902 (2002); private communications.

[10] F. S. Navarra et al., Phys. Lett. B 529, 87 (2002)

[11] B. Humpert and W. L. van Neerven, Phys. Lett. B 102, 426 (1981); Phys. Rev. D 25, 2593 (1982).

[12] T. H. Hansson, Su H. Lee and I. Zahed, Phys. Rev. D37, 2672 (1988).

[13] J. Bartels et al., hep-ph/9908411 (unpublished); J. Bartels and C. Bontus, Phys. Rev. D 61, 034009 (2000).

[14] S. Kim and Su H. Lee, Nucl. Phys. A679, 517 (2001).

[15] R. K. Ellis, W. Furmanski and R. Petronzio, Nucl. Phys. B207, 1 (1982); B212, 29 (1983).

[16] E. E. Salpeter and H. A. Bethe, Phys. Rev. 84, 1232 (1951).

[17] D. Ebert et al., Phys. Rev. D 62, 034014 (2000).

[18] A. D. Martin et al., Eur. Phys. J. C 4, 463 (1998).

[19] M. Glück et al., Z. Phys. C 67, 433 (1995).

[20] W. Lucha et al., Phys. Rev. D 46, 1088 (1992).

[21] L. Gerland et al., Phys. Rev. Lett. 81, 762 (1998) 